\title{
Identifying Autism Spectrum Disorder Symptoms Using Response and Gaze Behavior during the Go/NoGo Game CatChicken
}

Prasetia Utama Putra ( $\sim$ prasetia-putra-kt@ynu.jp )

Yokohama National University

Keisuke Shima

Yokohama National University

Koji Shimatani

Prefectural University of Hiroshima

Sergio A. Alvarez

Boston College

\section{Research Article}

Keywords: Gaze Modulation, Eye Tracker, Meta-learning Algorithm

Posted Date: July 13th, 2021

DOl: https://doi.org/10.21203/rs.3.rs-653887/v1

License: (c) This work is licensed under a Creative Commons Attribution 4.0 International License.

Read Full License

Version of Record: A version of this preprint was published at Scientific Reports on November 10th, 2021. See the published version at https://doi.org/10.1038/s41598-021-01050-7. 


\title{
Identifying Autism Spectrum Disorder Symptoms Using Response and Gaze Behavior during the Go/NoGo Game CatChicken
}

\author{
Prasetia Utama Putra ${ }^{1, *}$, Keisuke Shima $^{1}$, Sergio A. Alvarez ${ }^{2}$, and Koji Shimatani ${ }^{3}$ \\ ${ }^{1}$ Yokohama National University, Graduate School of Engineering, Japan \\ ${ }^{2}$ Boston College, Department of Computer Science, USA \\ ${ }^{3}$ Prefectural University of Hiroshima, Faculty of Health and Welfare, Japan
}

\begin{abstract}
Previous studies have found that Autism Spectrum Disorder (ASD) children scored lower during a Go/No-Go task and faced difficulty focusing their gaze on the speaker's face during a conversation. To date, however, there has not been an adequate study examining children's response and gaze during the Go/No-Go task to distinguish ASD from typical children. We investigated typical and ASD children's gaze modulation when they played a version of the Go/No-Go game. The proposed system represents the Go and the No-Go stimuli as chicken and cat characters, respectively. It tracks children's gaze using an eye tracker mounted on the monitor. Statistically significant between-group differences in spatial and auto-regressive temporal gaze-related features for 21 ASD and 31 typical children suggest that ASD children had more unstable gaze modulation during the test. Using the features that differ significantly as inputs, the AdaBoost meta-learning algorithm attained an accuracy rate of $88.6 \%$ in differentiating the ASD subjects from the typical ones.
\end{abstract}

\section{Introduction}

People often misinterpret invisible disorder symptoms in their children, such as inattentiveness and difficulty communicating with other people, as willful misconduct or misbehavior. The prevalence of clinical disorders, however, is high. In Japan, the prevalence of Autism Spectrum Disorder (ASD) symptoms among children has been estimated to be between 1.9\% and 9.3\% based on parent and teacher reports ${ }^{1}$; in the USA, about 1 of 54 children was diagnosed with ASD in $2020^{2}$, while in 2016, a study found that $9.41 \%$ children had Attention Deficit Hyperactive Disorder (ADHD) symptoms ${ }^{3}$.

Since the conventional diagnosis method requires comprehensive tests that are time-consuming, many studies have proposed to automatically distinguish ASD children from typical ones by utilizing machine learning with biosignals such as EEG ${ }^{4}$ or $\mathrm{fMRI}^{5}$. Nevertheless, those methods have been unable to provide a noninvasive system that can identify invisible disorder symptoms in early childhood.

In contrast, psychiatry studies recognize disorder symptoms by employing noninvasive behavioral tests, e.g., Go/ $\mathrm{NoGo}^{6-8}$ and visual attention tests. Previous studies have discovered a significant difference between ASD and typical children during a Go/NoGo task. The task requires a subject to react to the Go stimulus and inhibit their reaction to the NoGo stimulus ${ }^{8}$. The stimuli can be represented by visual objects with different colors and shapes ${ }^{6,8}$ or by sounds with different frequencies ${ }^{7}$. The task evaluates the subjects by measuring their percentages of correct responses and omission errors and their average response time and its variability. Children with ASD tend to perform worse ${ }^{9}$ and have high response time variability ${ }^{10}$ than typical children during the task, which may be caused by variability in neural activations ${ }^{11}$.

Moreover, studies on ASD children's gaze behavior have observed that the ASD group was slower to adjust their gaze to the stimulus position during eye-tracking measure of joint attention ${ }^{12}$, and faced difficulty in modulating their gaze during face-to-face conversation ${ }^{13}$. Previous works have found that temporal features of gaze are more informative than the global measurements in differentiating ASD from typically developing children. Swanson and Siller ${ }^{12}$ have found that ASD and typical children allocated the same amount of time at key areas but their duration of the first fixation to the target differed. Likewise, studies of gaze-shift ${ }^{14}$ and gaze-to-stimulus-distance ${ }^{15}$ have signified that gaze behavior of ASD and typical children differed significantly in the spatio-temporal aspect.

Although prior findings revealed a significant difference between typical and ASD children in performance on behavioral tests, it is unclear how to use those features in identifying disorder symptoms. This study aims to investigate the response and gaze behavior of children during the Go/NoGo task and to utilize features extracted from those measurements to identify ASD symptoms that suggest difficulty in inhibiting action and point of view ${ }^{16}$. Contrary to previous works on ASD subjects' gaze behavior, which have focused on global summary measures related to the gaze and stimulus positions, this study examined 
the intrinsic spatio-temporal structure of the gaze trajectories in greater detail by employing entropy-based and autoregressive features.

Using the CatChicken game ${ }^{17}$, we measured 21 high-risk and 31 typical children's response and gaze modulation; the use of a standardized task minimizes the bias that often occurs in face-to-face conversation. Spatial and gaze-adjustment features were extracted to represent each child's response, performance on the game, and gaze behavior. Statistical comparisons between typical and high-risk disorder children were performed using Student $t$ and Mann-Whitney $U$ tests $^{18}$. Additional details of the statistical methodology appear in the Methods section. The AdaBoost algorithm was employed ${ }^{19}$ to distinguish the features of ASD disorder children from those of typical children. Experiments employing spatial features, gaze-adjustment features, and a combination of them were conducted to identify differentiating features. Classification performance of the model was evaluated with accuracy, Matthews Correlation Coefficients (MCC) ${ }^{20}$, and Area Under the Curve (AUC) ${ }^{21}$ metrics and validated using three-fold cross-validation.

\section{Results}

\section{Spatial features}

Statistical analysis (Table 1) demonstrated a significant difference (by both Student $t$ and Mann-Whitney $U$ tests) between typical and ASD groups for eight spatial features: variance of fixation time, average and entropy of gaze acceleration, spectral entropy of gaze-to-object-distance, sample entropy of gaze distance, gaze angle, gaze-to-obj-distance, and velocity. For all such variables, the mean of the second group (ASD) was larger than that of the first group (typical); the corresponding effect sizes are large $\mathrm{e}^{22}(|d|>0.8)$, except for average acceleration, for which a moderately large effect size $(d=-0.763)$ was observed.

In contrast, although a medium effect size $(|d|>0.5)$ was observed for Go positive and negative percentages, response-time variance (RT-var), and gaze acceleration standard deviation, the differences between typical and ASD groups for those variables were insignificant. Nevertheless, within-group mean values indicated that ASD children more often responded incorrectly with higher response-time variance than the typical subjects.

Table 1

\begin{tabular}{|c|c|c|c|c|c|}
\hline & Student (p-value) & Whitney (p-value) & $d$ & Typical (mean \pm S.D.) & ASD (mean \pm S.D.) \\
\hline Go-positive & 0.042 & 0.040 & 0.591 & $30.8 \pm 9.9 \%$ & $24.2 \pm 12.9 \%$ \\
\hline Go-negative & 0.039 & 0.033 & -0.598 & $19.0 \pm 10.0 \%$ & $25.7 \pm 12.9 \%$ \\
\hline NoGo-positive & 0.712 & 0.404 & 0.105 & $46.2 \pm 2.7 \%$ & $45.9 \pm 3.4 \%$ \\
\hline NoGo-negative & 0.797 & 0.411 & -0.073 & $4.0 \pm 2.8 \%$ & $4.2 \pm 3.0 \%$ \\
\hline RT & 0.595 & 0.334 & 0.151 & $545 \pm 34$ & $539 \pm 49$ \\
\hline RT-var & 0.073 & 0.032 & -0.518 & $117 \pm 37$ & $136 \pm 36$ \\
\hline Trajectory-area & 0.845 & 0.321 & 0.056 & $0.521 \pm 0.103$ & $0.515 \pm 0.134$ \\
\hline Velocity-avg & 0.066 & 0.102 & -0.531 & $0.0088 \pm 0.0010$ & $0.0098 \pm 0.0024$ \\
\hline Velocity-var & 0.042 & 0.070 & -0.589 & $0.0176 \pm 0.0024$ & $0.0198 \pm 0.0051$ \\
\hline Acceleration-avg & $* 0.009$ & $* 0.002$ & -0.763 & $0.0035 \pm 0.0006$ & $0.0044 \pm 0.0016$ \\
\hline Acceleration-var & 0.022 & 0.035 & -0.668 & $0.0076 \pm 0.0015$ & $0.0092 \pm 0.0034$ \\
\hline Fixation-avg & 0.422 & 0.404 & 0.229 & $405 \pm 21$ & $399 \pm 29$ \\
\hline Fixation-var & $* 0.001$ & $* 0.001$ & -0.997 & $174 \pm 8.2$ & $183 \pm 8.3$ \\
\hline Distance-avg & 0.117 & 0.234 & -0.451 & $0.0160 \pm 0.0015$ & $0.0171 \pm 0.0035$ \\
\hline Distance-var & 0.095 & 0.136 & -0.482 & $0.0293 \pm 0.0033$ & $0.0316 \pm 0.0063$ \\
\hline Angle-avg & 0.292 & 0.500 & -0.301 & $0.0130 \pm 0.0013$ & $0.0136 \pm 0.0026$ \\
\hline Angle-var & 0.241 & 0.341 & -0.335 & $0.0285 \pm 0.0039$ & $0.0304 \pm 0.0073$ \\
\hline Distance-sen & $* 0.001$ & $* 0.001$ & -1.026 & $0.147 \pm 0.031$ & $0.202 \pm 0.076$ \\
\hline Angle-sen & $* 0.001$ & $* 0.001$ & -1.020 & $0.124 \pm 0.026$ & $0.169 \pm 0.063$ \\
\hline Velocity-sen & $* 0.000$ & $* \mathbf{0 . 0 0 0}$ & -1.133 & $0.137 \pm 0.034$ & $0.200 \pm 0.077$ \\
\hline Spatial-en & 0.993 & 0.195 & -0.002 & $0.894 \pm 0.011$ & $0.894 \pm 0.020$ \\
\hline Gaze-obj-en & $* 0.004$ & $* 0.004$ & -0.864 & $0.484 \pm 0.013$ & $0.497 \pm 0.017$ \\
\hline Gaze-obj-sen & $* 0.001$ & $* 0.001$ & -0.973 & $0.130 \pm 0.018$ & $0.153 \pm 0.031$ \\
\hline Gaze-obj-spe & $* 0.003$ & $* 0.002$ & -0.880 & $0.354 \pm 0.008$ & $0.361 \pm 0.006$ \\
\hline
\end{tabular}




\section{Gaze-adjustment features}

A significant difference between the groups was observed in the mean values of $\alpha$ and $\theta_{2}$ by the Mann-Whitney $U$ test (Table 2). In contrast, both the Student $t$ test and effect size $(|d|<0.2)$ suggested that ASD children's gaze-adjustment features did not differ from the typical ones.

Separating gaze-adjustment features according to response types (Go-positive, Go-negative, NoGo-positive, and NoGonegative) yielded statistically significant differences between typical and ASD children in all auto-regressive coefficients by the Mann-Whitney $U$ test, as well as greater effect size (mean $|d|>0.4$ ). The t-test results also signified that ASD gaze modulation differed when they responded incorrectly to the Go stimulus and correctly to the NoGo stimulus.

Furthermore, extrapolation of the gaze-to-obj distance in time using the average values of the autoregressive coefficients suggests that separating the features (Fig. 1C - J) produces a more obvious difference between the groups than mixing them (Fig. 1A, B). Typical children adjusted their gaze to the stimulus position faster when they responded correctly to the Go and NoGo characters and when they reacted incorrectly to the latter stimulus (Fig. 1C, G, I); the velocity of their extrapolated gaze-adjustment (Fig. 1D, H, J) was \pm 0.0014 faster compared to the ASD children (the velocity of extrapolated gaze-adjustment was computed by averaging the negative of the first derivative of the extrapolated gaze-to-obj distance over time). Nevertheless, typical children modulated their gaze in a similar way to the ASD subjects when they missed the Go stimulus (Fig. 1E, F).
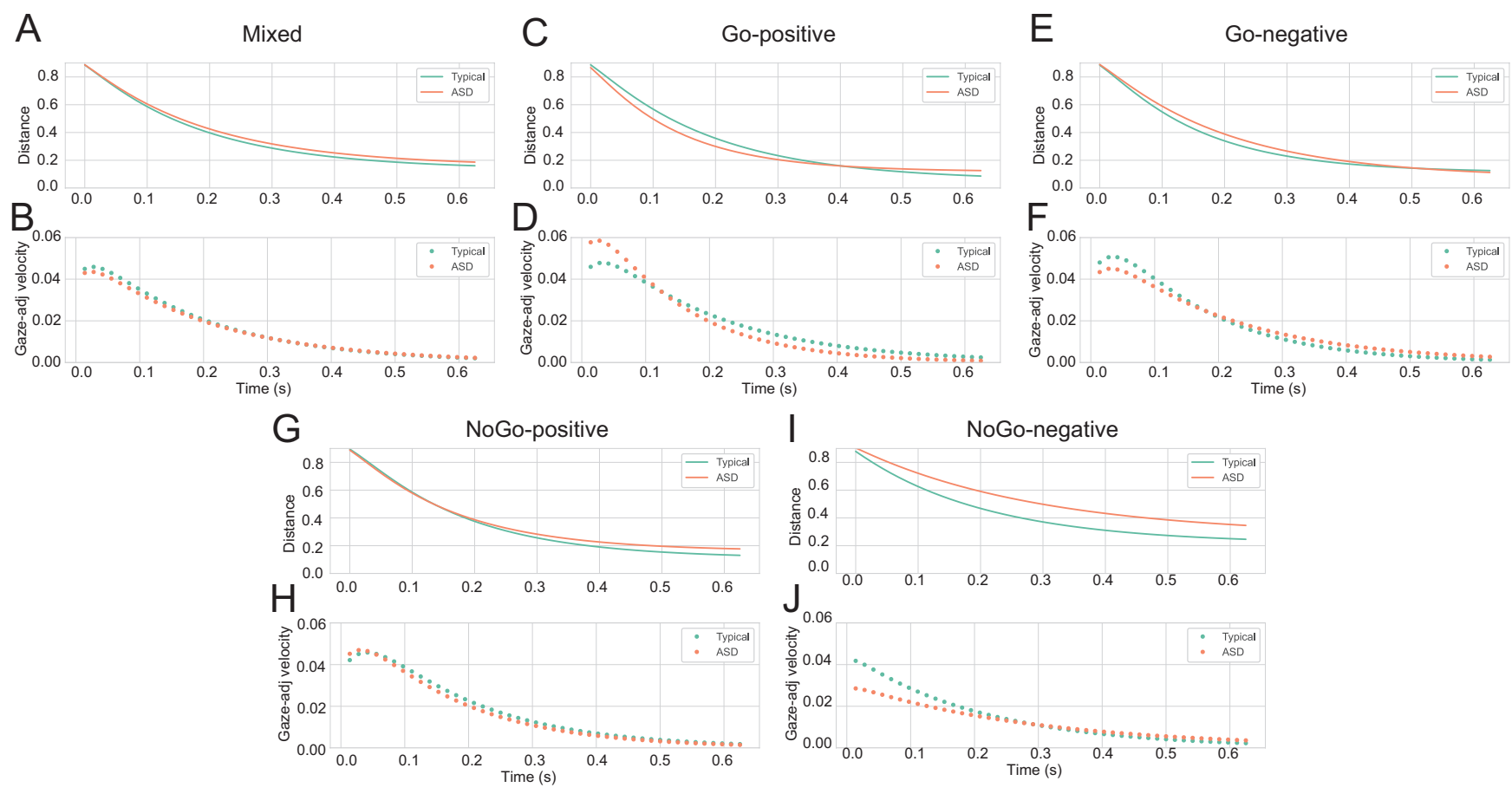

Figure 1

\section{Classification}

Classification results (Table 3) showed that when using only spatial features, the accuracy of the AdaBoost model was $6.1 \%$ lower than when utilizing gaze-adjustment features. A significant increase in the model's recognition rate occurred when separating the gaze-adjustment features based on response types (response-type-gaze features). The classification rate was $17.1 \%$ higher than when employing gaze-adjustment features.

Using both response-type-gaze and significant spatial features, the model obtained an insignificant increase in its accuracy rate, which was $0.3 \%$ higher than when using the former features alone. Combining the gaze features with significant spatial and game performance features, however, decreased the accuracy rate by $4.1 \%$.

The MCC score agreed with the accuracy results: combining response-type-gaze features and significant spatial features yielded a 0.01 higher MCC score than using only response-type-gaze features. Even though the increase was insignificant, the high MCC score indicated that the model's prediction results strongly correlated with the ground-truth labels, and the model could reliably recognize both the typical and ASD children. Besides, the AdaBoost obtained AUC scores higher than 0.85 when utilizing response-type-gaze features and when combining them with significant spatial and performance features. This suggested that high performance could be expected from the algorithm when employing those features ${ }^{23}$ 
Table 2

\begin{tabular}{|l|r|r|r|r|r|}
\hline & Student (p-value) & Whitney (p-value) & $d$ & Typical (mean \pm S.D.) & ASD (mean \pm S.D.) \\
\hline Mixed & & & & & \\
\hline$\alpha$ & 0.646 & $* \mathbf{0 . 0 0 0}$ & -0.065 & $0.004 \pm 0.012$ & $0.005 \pm 0.013$ \\
\hline$\theta_{1}$ & 0.282 & $* \mathbf{0 . 0 2 3}$ & 0.153 & $1.488 \pm 0.221$ & $1.456 \pm 0.189$ \\
\hline$\theta_{2}$ & 0.272 & $* \mathbf{0 . 0 0 0}$ & -0.156 & $-0.520 \pm 0.206$ & $-0.489 \pm 0.191$ \\
\hline Go-positive & & & & & \\
\hline$\alpha$ & 0.180 & $* \mathbf{0 . 0 0 0}$ & -0.385 & $0.002 \pm 0.009$ & $0.005 \pm 0.191$ \\
\hline$\theta_{1}$ & 0.185 & $* \mathbf{0 . 0 2 3}$ & 0.379 & $1.533 \pm 0.060$ & $1.488 \pm 0.167$ \\
\hline$\theta_{2}$ & 0.391 & $* \mathbf{0 . 0 0 0}$ & -0.245 & $-0.561 \pm 0.054$ & $-0.529 \pm 0.193$ \\
\hline Go-negative & & & & & \\
\hline$\alpha$ & 0.206 & $* \mathbf{0 . 0 0 0}$ & 0.362 & $0.003 \pm 0.002$ & $0.002 \pm 0.005$ \\
\hline$\theta_{1}$ & $* \mathbf{0 . 0 1 6}$ & $* \mathbf{0 . 0 0 0}$ & 0.703 & $1.572 \pm 0.059$ & $1.521 \pm 0.084$ \\
\hline$\theta_{2}$ & $* \mathbf{0 . 0 0 6}$ & $\boldsymbol{* 0 . 0 0 0}$ & -0.807 & $-0.601 \pm 0.054$ & $-0.549 \pm 0.077$ \\
\hline NoGo-positive & & & & & \\
\hline$\alpha$ & 0.135 & $* \mathbf{0 . 0 0 0}$ & -0.429 & $0.003 \pm 0.002$ & $0.005 \pm 0.008$ \\
\hline$\theta_{1}$ & $* \mathbf{0 . 0 0 0}$ & $* \mathbf{0 . 0 2 3}$ & 1.155 & $1.606 \pm 0.031$ & $1.540 \pm 0.081$ \\
\hline$\theta_{2}$ & $* \mathbf{0 . 0 0 0}$ & $* \mathbf{0 . 0 0 0}$ & -1.259 & $-0.631 \pm 0.028$ & $-0.571 \pm 0.066$ \\
\hline NoGo-negative & & & & & \\
\hline$\alpha$ & 0.877 & $* \mathbf{0 . 0 0 0}$ & 0.044 & $0.010 \pm 0.021$ & $0.009 \pm 0.023$ \\
\hline$\theta_{1}$ & 0.688 & $* \mathbf{0 . 0 0 0}$ & -0.114 & $1.241 \pm 0.321$ & $1.274 \pm 0.238$ \\
\hline$\theta_{2}$ & 0.794 & $* \mathbf{0 . 0 0 0}$ & 0.074 & $-0.286 \pm 0.295$ & $-0.306 \pm 0.230$ \\
\hline
\end{tabular}

Table 3

\begin{tabular}{|l|r|r|r|}
\hline Modality & Accuracy (mean \pm S.D.) & MCC (mean \pm S.D.) & AUC (mean \pm S.D.) \\
\hline Spatial & $65.1 \pm 8.9$ & $0.29 \pm 0.17$ & $0.67 \pm 0.09$ \\
\hline Gaze-adjustment & $71.2 \pm 7.9$ & $0.40 \pm 0.16$ & $0.79 \pm 0.11$ \\
\hline Response-type-gaze & $88.3 \pm 4.9$ & $0.79 \pm 0.08$ & $0.95 \pm 0.02$ \\
\hline Response-type-gaze+significant & $88.6 \pm 4.4$ & $0.80 \pm 0.07$ & $0.90 \pm 0.03$ \\
\hline Response-type-gaze+significant+performance & $84.4 \pm 10.1$ & $0.71 \pm 0.19$ & $0.90 \pm 0.01$ \\
\hline
\end{tabular}

The confusion matrix (Table 4), however, shows that the model more frequently misclassified typical subjects as ASD (false-positive) than it misclassified ASD subjects as typical (false-negative). Visualization of the features through star plots (Fig. 2) reveals higher mean values and variability in the misclassified typical subjects' features than the correctly-classified subjects'. On the other hand, the features of misclassified ASD subjects show lower mean values and variability.

Table 4

\begin{tabular}{|l|l|l|}
\cline { 2 - 3 } \multicolumn{1}{c|}{} & $\begin{array}{l}\text { Predicted: } \\
\text { Typical }\end{array}$ & $\begin{array}{l}\text { Predicted: } \\
\text { ASD }\end{array}$ \\
\hline $\begin{array}{l}\text { Actual: } \\
\text { Typical }\end{array}$ & $83.9 \%$ & $16.1 \%$ \\
\hline $\begin{array}{l}\text { Actual: } \\
\text { ASD }\end{array}$ & $4.8 \%$ & $95.2 \%$ \\
\hline
\end{tabular}

\section{Discussion}

This study evaluated whether features extracted from response and gaze behavior during Go/NoGo task can be used to identify ASD symptoms in children. We utilized the CatChicken game ${ }^{17}$ to measure the response and gaze modulation of 21 ASD and 31 typical children. During the game, the children should respond to the chicken character (Go stimulus) by pressing a space bar but should inhibit their action towards the cat character (NoGo stimulus). 
A

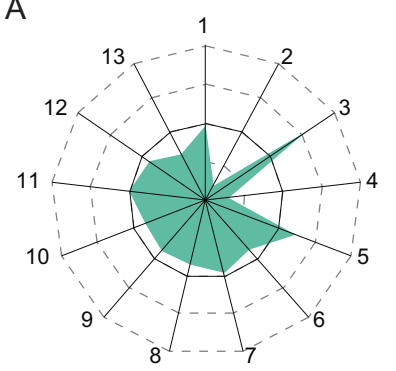

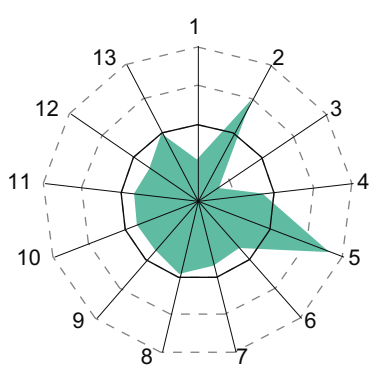

Correctly-classified typical children

C

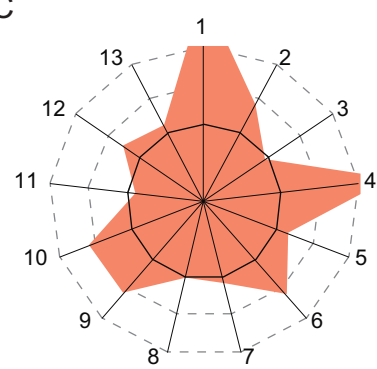

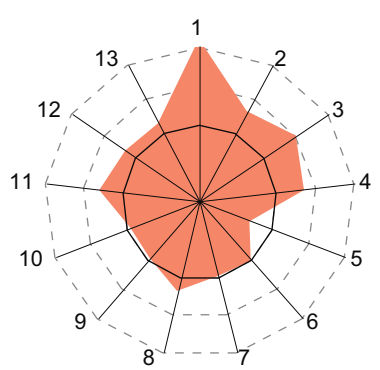

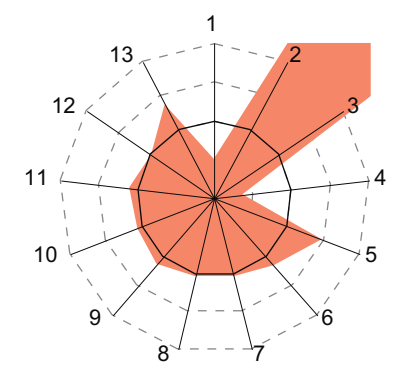

B

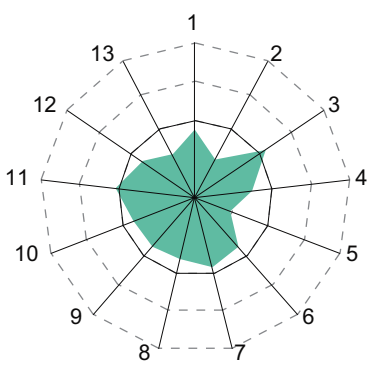

B

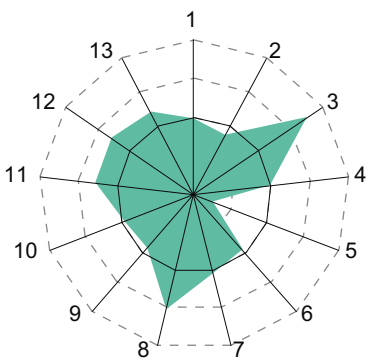

Misclassified typical child

D

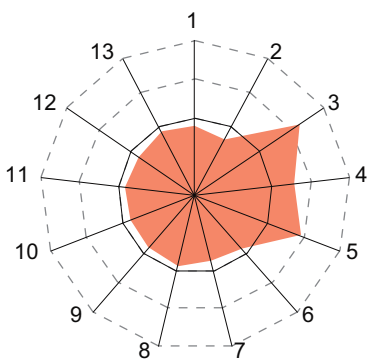

Misclassified ASD child

Figure 2

The game outputs four variables: response types and times, and the stimulus and gaze locations over time. Statistical analysis using Student $t$ and Mann-Whitney $U$ tests were performed on spatial and gaze-adjustment features extracted from those variables.

As we expected, we found a significant difference in gaze modulation between ASD and typical children. Previous studies found that ASD children's gaze movement differed significantly from typical children's in terms of variability of the gaze pattern $^{24}$, the fixation time spent on the stimulus ${ }^{13}$, and duration of the first fixation to the target ${ }^{12}$. Our results suggest lower accuracy and greater randomness of the ASD subjects' visual tracking of the target: the relative gaze-to-object difference was less steady over time than for typical subjects, and predictability of ASD subjects' gaze was lower as measured by sample entropy of both distance and angle. A greater irregularity of gaze distance and angle may indicate that ASD children over-interpreted the information of a given stimulus, thereby causing more unintentional viewing behavior ${ }^{25}$. The higher value of ASD children's gaze-to-object entropy suggested less structured tracking in a spatial sense, while a greater value of sample entropy value demonstrated lowered predictability of the gaze-to-object difference as a function of time. Likewise, greater spectral entropy indicated less structure of the frequency content of ASD subjects' gaze signals.

Second, statistical analysis using the Mann-Whitney $U$ test demonstrated significant differences in the gaze-adjustment features between the groups. The extrapolation results show that ASD children, on average, adjusted their gaze more slowly to the stimulus location than their typical peers. When ASD children reacted incorrectly towards the stimulus, their extrapolation results tended to be slower at the beginning. The results, however, did not signify that typical subjects' gaze movement was faster, as an insignificant difference was observed in gaze velocity between the groups.

In contrast, gaze trajectory area and the entropy of gaze distribution showed no significant differences between typical and ASD populations. While our previous work ${ }^{17}$ found greater dispersion of gaze movement in ASD children, the results of the present paper suggest that global measures of gaze behavior are similar in the two groups. The discrepancy may be due to the greater size of the sample available for the present paper. Swanson and Siller ${ }^{12}$ also observed that total gaze allocation of ASD and typical children did not differ but their temporal gaze movement (duration of the first fixation to the target) did. Our present findings and theirs provide compelling evidence that the gaze behavior of ASD children may differ from the typically developing children in the temporal aspect.

Another major finding of this study was that the performance of the game and response time of ASD and typical children did not differ significantly. Even though we observed greater Go and NoGo negative percentages and higher response time variance in ASD children, statistical analysis demonstrated an insignificant difference between the groups. The results contradict previous works that observed greater RT variability of go-response ${ }^{9}$ and higher omission error ${ }^{10}$ in ASD population than their typical peers. One interpretation of these findings is that the insignificant difference of RT was because this work computed RT 
of both Go and NoGo trials; Lee et.al ${ }^{26}$ found similar RT for ASD and typical subjects. Outlier removal in the pre-processing step of the present work might affect the statistical results of game performance and RT variability, as well.

Lastly, our classification results suggest promising performance of more detailed spatio-temporal features extracted from children's gaze during the Go/NoGo task than those used in prior work. Furthermore, our classification results show that using responses and gaze features together yields a higher recognition rate in differentiating ASD from typical children than using either type of information alone.

\section{Conclusion}

This study examined the difference in gaze behavior and response features of ASD and typical children during the Go/NoGo task. In contrast to our hypothesis, the experimental results suggest that using gaze behavior features alone yielded higher performance in differentiating ASD from typical children than combining them with features extracted from participant's responses

Two limitations of this work are the relatively small sample size and the limited number of features. This study involved 22 ASD and 35 typical children with a narrow age range and found that among these subjects only one gaze modulation existed: all subjects adjusted their gaze to the stimulus position. Results of our previous work ${ }^{17}$, however, suggested that among older subjects there were two types of viewing behavior: the ones who adjusted their gaze (55.9\% of total subjects) and the one who concentrated it on the middle of the screen (44.1\% of the total subjects). Also, since this work only measured response behavior by calculating response time and game performance, which represented the execution stage of responses, the difference between groups in the preparation stage of responses is unclear. Future studies should involve more subjects with a wide range of age and measure both preparation and execution stages of participants' responses.

\section{Methods}

\section{Catchicken game}

The Catchicken ${ }^{17}$ game was utilized to measure children's response and gaze movement during a Go/NoGo task. The Go/NoGo task was used to measure a person's inhibitory control; a subject should respond to the Go stimulus but inhibit their action towards the NoGo stimulus 8 . The game represented the Go and NoGo stimuli as "Chicken" and "Cat" characters, respectively. A stimulus appeared randomly in one of nine locations for a fixed duration of time (Fig. 3). The interval between two consecutive stimuli was set by configuring the minimum and maximum waiting-time values.

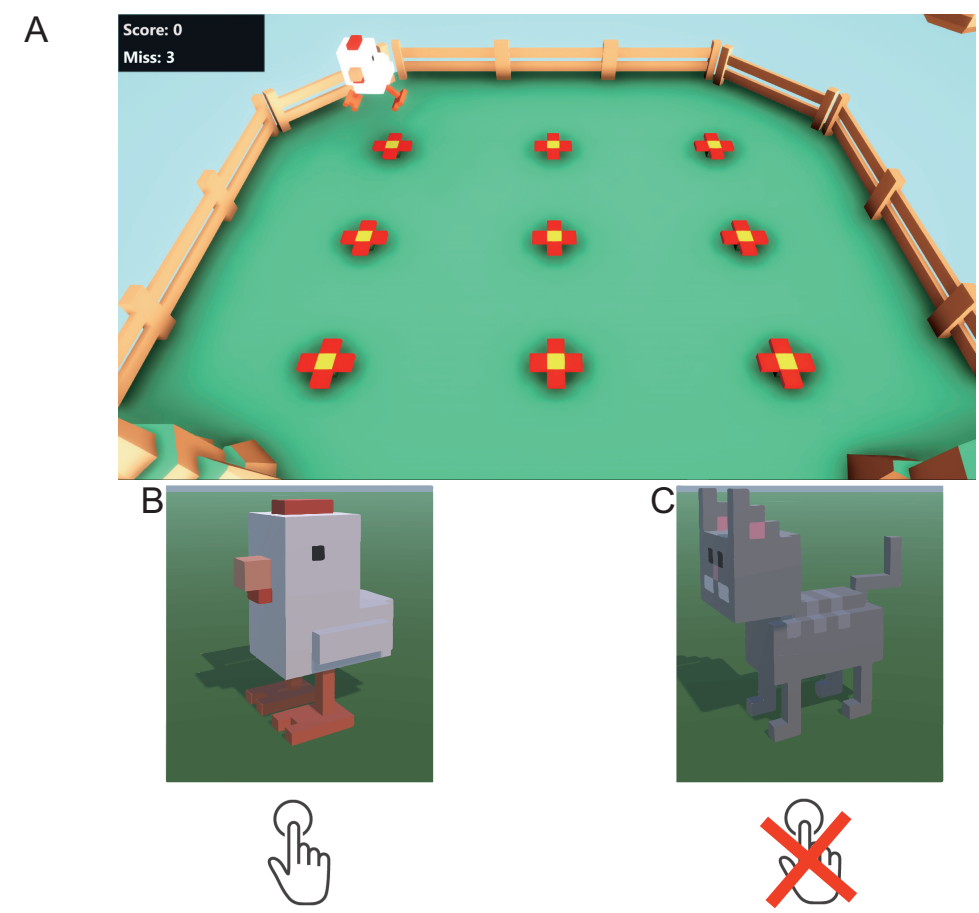

Figure 3 
The system outputted the user's response types and time, and stimulus and eye locations on the monitor (Fig. 4). A user responded to the stimulus by pressing the spacebar. The system categorized a subject's response as one of four types: Go-positive if the subject responded to the Go character; Go-negative if he missed it; NoGo-positive if he inhibited his action in response to the NoGo character; NoGo-negative if he reacted to it. Different sound feedback was given when the subject responds correctly and incorrectly towards the stimulus. The system was equipped with a Tobii $4 \mathrm{C}$ eye tracker that recorded the user's eye position on the monitor continuously. The eye tracker sampling rate was $90 \mathrm{~Hz}$ (interlaced), and its operating distance was $50 \mathrm{~cm}$ to $95 \mathrm{~cm}$. The stimulus and eye locations on the monitor were normalized to the unit interval [0,1] by dividing the pixel coordinates by the window's coordinate length.

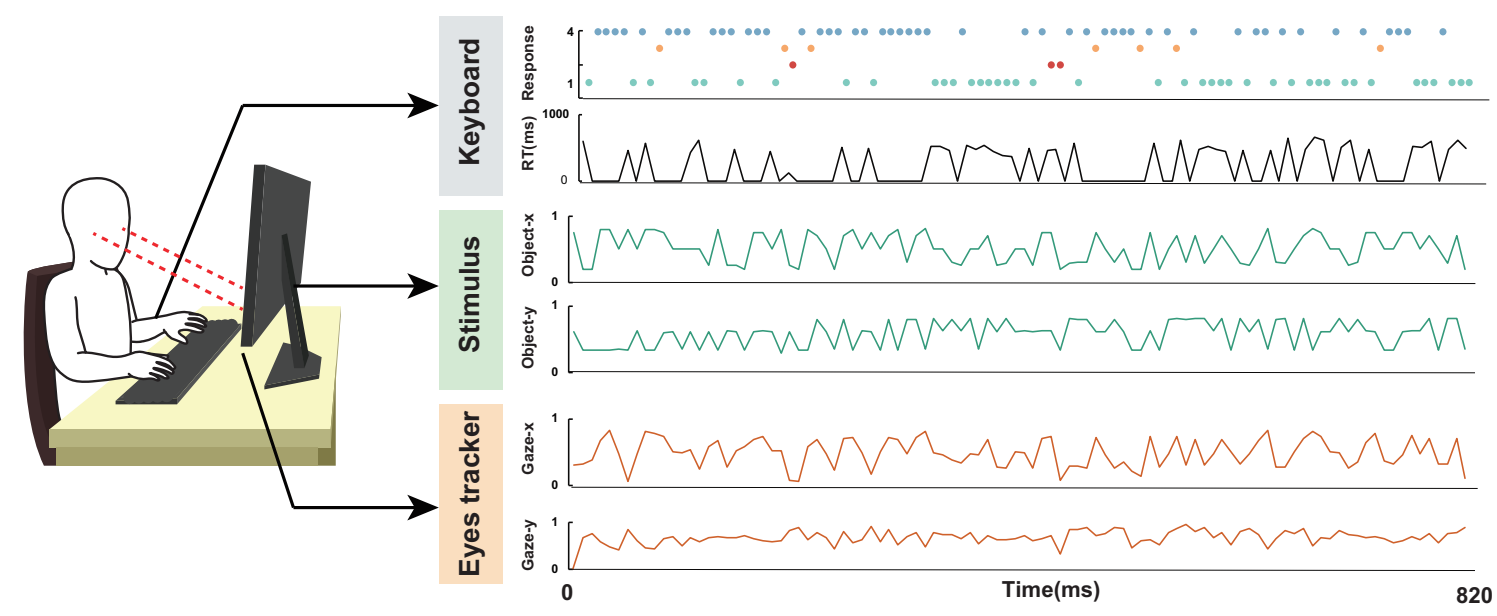

Figure 4

\section{Participants}

Participants involved 22 autism spectrum disorder children (16 male and 6 female) and 35 typical children (24 male and 11 female) with an average age of five years from two local schools in Japan. All ASD subjects attended special education school and had been diagnosed by clinicians; 11 ASD children also had attention deficit symptoms and seven of them were diagnosed as hyperactive as well. Both ASD and typical children did not have any physical disorder. One ASD child (male) and four typical children ( 1 male and 3 female) were excluded because their data were corrupted. Therefore, this study only processed 21 ASD and 31 typical children's data.

During the experiment, the subjects were seated in front of a notebook equipped with an eye tracker and web camera (Fig. 5). They responded to the stimulus by pressing the spacebar on the keyboard. The proportion of the Go and NoGo stimuli was uniform and the order of appearance was set in advance; their appearance time was $700 \mathrm{~ms}$; the minimum and maximum of the waiting period were 700 and $1000 \mathrm{~ms}$.

Before starting the experiment, the eye tracker was calibrated and an instructor explained the game and its rules to the subject. All subjects participated in a one-minute training session before taking a four-minute evaluation.

\section{Ethics statement and consent}

Before participating in the experiment, informed consent was obtained from teachers and parents on behalf of the children. The study was approved by the Research Ethics Committee of the Prefectural University of Hiroshima (letter no: 15MH070) and was conducted in accordance with the amended Declaration of Helsinki.

\section{Features}

Figure 6 shows the pipeline for extracting spatial and gaze-adjustment features from response and gaze data. Before extracting the features, preprocessing was performed to eliminate noise and redundant data. The responses whose RT was less than a threshold were considered as outliers; $6.6 \%$ of typical and 7.3\% of ASD data were removed. The threshold was the RT's median absolute deviation ${ }^{27}$ (104.75 ms) multiplied by a constant scale factor of the normal distribution $(1.4826)$ : $1.4826 \times$ $104.75=155.30 \mathrm{~ms}$. The data were down-sampled from $144 \mathrm{~Hz}$ to $72 \mathrm{~Hz}$ to remove redundancy; a Savitzky-Golay filter ${ }^{28}$ ( $\mathrm{n}=5$ and poly=2) was used to perform smoothing to prevent artifacts during numerical differentiation.

\section{Spatial features}

The spatial features comprised 24 attributes (Table 5) that can be grouped into three categories: game performance, absolute gaze position, and gaze-to-object movement. 


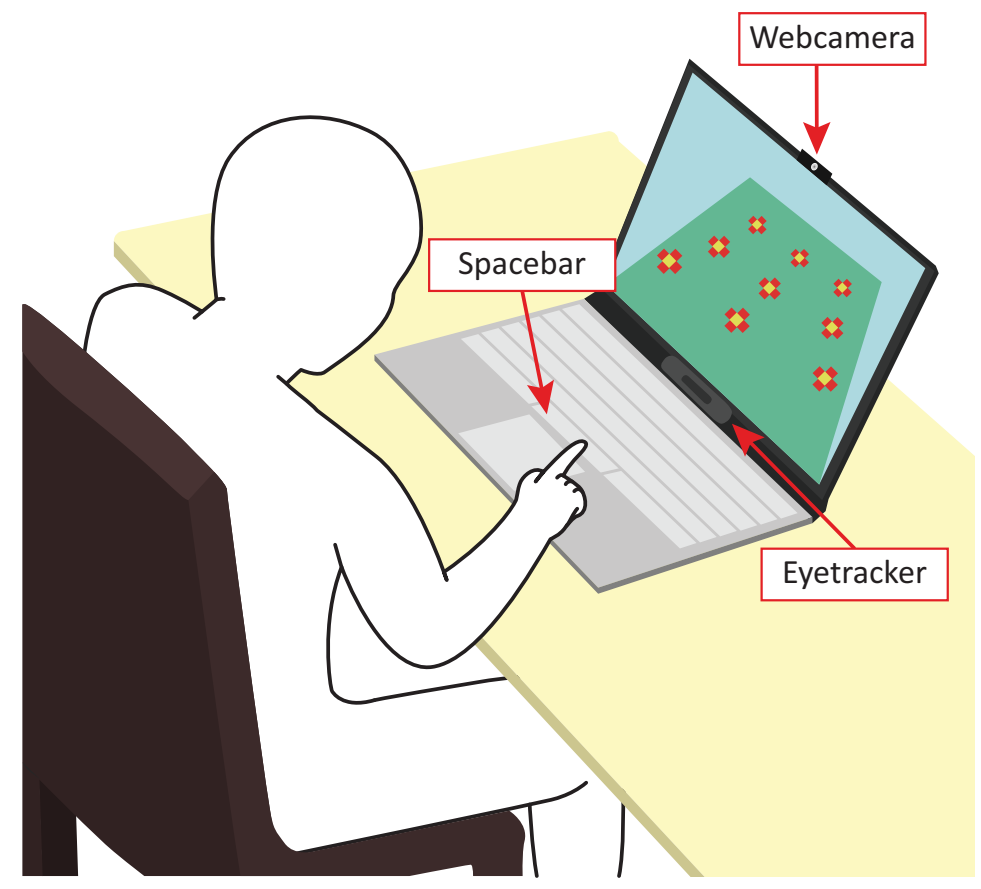

Figure 5

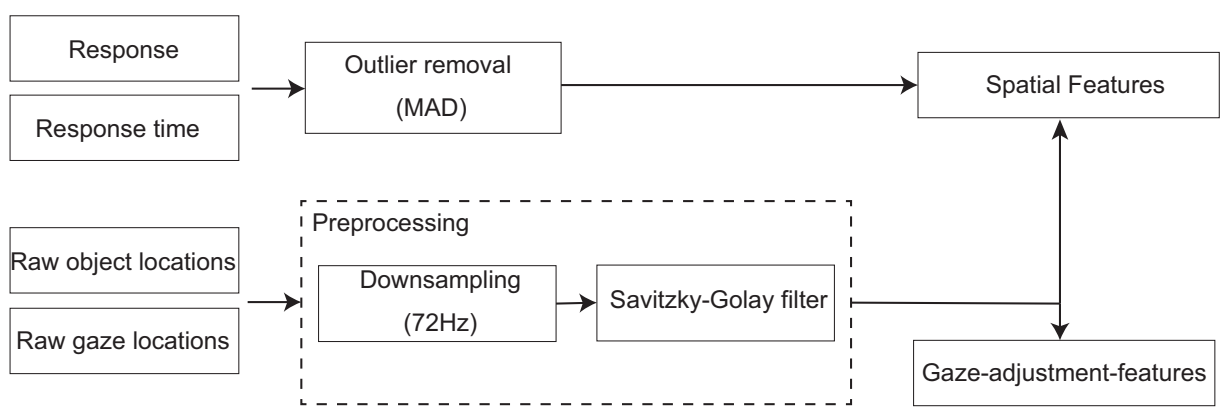

Figure 6

Game performance features consisted of subjects' response time (RT) and variance (RT-var) and the percentages of their positive and negative responses towards the Go and NoGo stimuli. RT (equation (1)) measured the time difference between when the character appeared $\left(t_{a}\right)$ and when the subject reacted to it $\left(t_{r}\right)$; while RT-var was the standard deviation of RT. The RT of the Go and NoGo stimuli were not separated.

$$
R_{t}=t_{a}-t_{r}
$$

Gaze position features measured the absolute position of subjects' gaze during the experiment. The gaze trajectory area was calculated using the Convex Hull algorithm ${ }^{29}$, and its value was normalized into 0 to 1 . Gaze velocity and acceleration along the $x$ and $y$ axes were calculated respectively as the Savitzky-Golay smoothed first and second time-derivatives of the corresponding coordinate locations. Gaze distance (equation (4)) and angles (equation (5)) were the Euclidean distance and angle between gaze positions $(g(t)$ and $g(t+1))$. The gaze spatial distribution was estimated using a 2D histogram algorithm, using 100 cells along each of the $x$ and $y$ axes.

$$
v=\sqrt{\partial_{t} x^{2}+\partial_{t} y^{2}}
$$




$$
\begin{aligned}
& \partial v=\sqrt{\partial_{t}^{2} x^{2}+\partial_{t}^{2} y^{2}} \\
& d(t)=|g(t+1)-g(t)| \\
& a(t)=\arccos \left(\frac{g(t+1) \cdot g(t)}{|g(t+1)| \cdot|g(t)|}\right)
\end{aligned}
$$

Gaze-to-object movement comprised the features that computed the relative position between subjects' gaze position and the stimulus position. Fixation time was measured as the time difference between when the subject's gaze entered and when it left the stimulus area; the area was a circle of radius 0.25 (measured by Euclidean distance) from the center of the stimulus. Gaze-to-object difference was subjects' gaze positions minus object positions when the latter appeared on the screen. Kernel density estimation and Welch's method ${ }^{30}$ (nperseg=32) were employed to estimate the probability and spectral densities of the difference, respectively. The probability distribution of the gaze-to-object difference was computed using 50 cells along each of the $x$ and $y$ axes.

Regularity of gaze distribution was computed using Shannon entropy ${ }^{31}$ and expressed as:

$$
H_{s}=-\int p(x, y) \log _{2}(p(x, y)) d x d y
$$

where $p(x, y)$ was the probability density of a state in a two-coordinate plane; while for power spectral density, $p(x, y)$ was the sum of squared magnitudes of the Fourier transforms of the respective $\mathrm{x}$ and y components. Greater entropy indicates less intentional viewing behavior ${ }^{25}$ and suggests greater gaze dispersion. The final value of gaze entropy was normalized by diving by the maximum possible entropy $\log _{2}(N)$, in which $N$ was the total number of states; in this study, $\mathrm{N}$ equaled to the total number of cells along the $x$ and $y$ axes.

Temporal randomness of gaze movement was measured by sample entropy (sen). Sen is equal to the negative natural logarithm of the probability that two subsequences of equal length $m$ that are similar will remain similar at the next time step ${ }^{32}$; higher sample entropy means lower predictability within the original sequence. This study set $m$ to two and estimated the distance between two template vectors with Chebyshev distance.

\section{Gaze-adjustment features}

Gaze-adjustment measured the distance between participants' gaze and stimulus positions during times when the stimulus was presented onscreen. Euclidean distance was used as the distance metric, with values ranging from 0 to $\sqrt{2}$. Since the appearance time of each stimulus depended on the subjects' RT, which varied, each gaze-adjustment was represented by auto-regressive parameters (equation (7)). The model's lag $L$ was set to two (average of AIC: -9.129 ); hence, each gaze-adjustment was represented by three variables: $\alpha, \theta_{2}$, and $\theta_{1}$.

$$
y_{t}=\alpha+\sum_{i=1}^{L} \theta_{i} y_{t-i}
$$

This study computed the average value of each coefficient for typical and ASD groups using the arithmetic means over the respective groups. Also, the average coefficients were calculated for each response type to investigate the difference between ASD and typical children's gaze adjustment characteristics in more detail.

\section{Data analyses}

\section{Statistical analysis}

The comparison between groups was computed using Student $t$ and Mann-Whitney $U^{18}$ tests. The effect size was calculated with Cohen's $d^{33}$; typical and ASD subjects were treated as the first and the second groups, respectively. The Benjamini-Hochberg procedure $^{34}$ was utilized to control the false discovery rate associated with multiple comparisons, at the level 0.05. 
Table 5

\begin{tabular}{|l|l|}
\hline$\#$ & Detail \\
\hline Go-positive & The percentage of Go response \\
\hline Go-negative & The percentage of Go-negative response \\
\hline NoGo-positive & The percentage of NoGo response \\
\hline NoGo-negative & The percentage of NoGo-negative response \\
\hline RT & The average of a subject response time \\
\hline RT-var & The standard deviation of a subject response time \\
\hline Trajectory-area & The gaze trajectory area \\
\hline Velocity-avg & The average velocity of subjects' gaze \\
\hline Velocity-var & The standard deviation of the velocity of subjects' gaze \\
\hline Acceleration-avg & The average acceleration of subjects' gaze \\
\hline Acceleration-var & The standard deviation of the velocity of subjects' gaze along the y-axis \\
\hline Fixation-avg & The average of subjects' fixation time \\
\hline Fixation-var & The standard deviation of subjects' fixation time \\
\hline Distance-avg & The average of gaze distance \\
\hline Distance-var & The standard deviation of gaze distance \\
\hline Angle-avg & The average of gaze angle \\
\hline Angle-var & The standard deviation of gaze angle \\
\hline Distance-sen & Sample entropy of subjects' gaze distance \\
\hline Angle-sen & Sample entropy of subjects' gaze angle \\
\hline Velocity-sen & Sample entropy of gaze velocity \\
\hline Spatial-en & The entropy of subjects' gaze distribution \\
\hline Gaze-obj-en & The entropy of the difference between subjects' gaze and stimulus position \\
\hline Gaze-obj-sen & Sample entropy of the Euclidean distance between subjects' gaze and stimulus position \\
\hline Gaze-obj-spe & Spectral entropy of the difference between subjects' gaze and stimulus position \\
\hline
\end{tabular}

\section{Classification}

Classification was performed to verify how informative the spatial and gaze-adjustment features are for differentiating ASD children from the typical ones. Instead of using raw gaze-adjustment features, this study computed the gaze-adjustment features' skewness, kurtosis, average, and standard deviation. Then, the features' dimension was reduced with Neighborhood Component Analysis $^{35}$ and five components were retained; Principal Component Analysis was employed to initialize the transformation.

Spatial and gaze-adjustment features were normalized using $z$-normalization. The AdaBoost ${ }^{19}$ with decision tree base estimators was employed to distinguish the features of ASD children from those of typical children. The model's hyperparameter values were optimized using a grid search algorithm. In particular, this study validated the max-depth of the estimator in the set $\{1,3,5,7\}$, the max-leaf of the estimator in $\{3,5,7\}$, the number of estimators in $\{15,25,50,75\}$, and the AdaBoost's learning-rate in $\{0.5,0.75\}$. The max-feature-proportion of the decision tree was set to 0.5 for higher and lower proportion decreased the overall accuracy performance.

Accuracy, the Matthews Correlation Coefficient (MCC) ${ }^{20}$, and Area Under the Curve (AUC) ${ }^{21}$ were used as classification performance metrics. The decision threshold for classification was set to 0.5 , thereby predicted probability greater or equal to 0.5 was converted to ASD class and typical class, otherwise. Three-fold cross-validation was used to validate the model.

\section{References}

1. Chiarotti, F. \& Venerosi, A. Epidemiology of autism spectrum disorders: A review of worldwide prevalence estimates since 2014. Brain Sci. 10, 274-294 (2020).

2. Maenner, M. J., Shaw, K. A., Baio, J. et al. Prevalence of autism spectrum disorder among children aged 8 years-autism and developmental disabilities monitoring network, 11 sites, united states, 2016. MMWR Surveillance Summ. 69, 1 (2020).

3. Danielson, M. L. et al. Prevalence of parent-reported adhd diagnosis and associated treatment among us children and adolescents, 2016. J. Clin. Child \& Adolesc. Psychol. 47, 199-212 (2018).

4. Mohammadi, M. R. et al. Eeg classification of adhd and normal children using non-linear features and neural network. Biomed. Eng. Lett. 6, 66-73 (2016). 
5. Peng, X., Lin, P., Zhang, T. \& Wang, J. Extreme learning machine-based classification of adhd using brain structural mri data. PloS one 8, e79476 (2013).

6. Bezdjian, S., Baker, L. A., Lozano, D. I. \& Raine, A. Assessing inattention and impulsivity in children during the go/nogo task. Br. J. Dev. Psychol. 27, 365-383 (2009).

7. Kropotov, J. D. et al. Erps correlates of eeg relative beta training in adhd children. Int. journal psychophysiology 55, 23-34 (2005).

8. Eagle, D. M., Bari, A. \& Robbins, T. W. The neuropsychopharmacology of action inhibition: cross-species translation of the stop-signal and go/no-go tasks. Psychopharmacology 199, 439-456 (2008).

9. Magnuson, J. R., Iarocci, G., Doesburg, S. M. \& Moreno, S. Increased intra-subject variability of reaction times and single-trial event-related potential components in children with autism spectrum disorder. Autism Res. 13, 221-229 (2020).

10. Uzefovsky, F., Allison, C., Smith, P. \& Baron-Cohen, S. Brief report: the go/no-go task online: inhibitory control deficits in autism in a large sample. J. Autism Dev. Disord. 46, 2774-2779 (2016).

11. Müller, R.-A., Kleinhans, N., Kemmotsu, N., Pierce, K. \& Courchesne, E. Abnormal variability and distribution of functional maps in autism: an fmri study of visuomotor learning. Am. J. Psychiatry 160, 1847-1862 (2003).

12. Swanson, M. R. \& Siller, M. Patterns of gaze behavior during an eye-tracking measure of joint attention in typically developing children and children with autism spectrum disorder. Res. Autism Spectr. Disord. 7, 1087-1096 (2013).

13. Noris, B., Nadel, J., Barker, M., Hadjikhani, N. \& Billard, A. Investigating gaze of children with asd in naturalistic settings. PloS one 7, e44144 (2012).

14. Falck-Ytter, T., Fernell, E., Hedvall, Å. L., Von Hofsten, C. \& Gillberg, C. Gaze performance in children with autism spectrum disorder when observing communicative actions. J. autism developmental disorders 42, 2236-2245 (2012).

15. Falck-Ytter, T., von Hofsten, C., Gillberg, C. \& Fernell, E. Visualization and analysis of eye movement data from children with typical and atypical development. J. autism developmental disorders 43, 2249-2258 (2013).

16. Baron-Cohen, S., Wheelwright, S., Skinner, R., Martin, J. \& Clubley, E. The autism-spectrum quotient (aq): Evidence from asperger syndrome/high-functioning autism, malesand females, scientists and mathematicians. J. autism developmental disorders 31, 5-17 (2001).

17. Putra, P., Shima, K. \& Shimatani, K. Catchicken: A serious game based on the go/nogo task to estimate inattentiveness and impulsivity symptoms. In 2020 IEEE 33rd International Symposium on Computer-Based Medical Systems (CBMS), 152-157 (IEEE, 2020).

18. Mann, H. B. \& Whitney, D. R. On a test of whether one of two random variables is stochastically larger than the other. The annals mathematical statistics 50-60 (1947).

19. Freund, Y. \& Schapire, R. E. A decision-theoretic generalization of on-line learning and an application to boosting. J. computer system sciences 55, 119-139 (1997).

20. Matthews, B. W. Comparison of the predicted and observed secondary structure of t4 phage lysozyme. Biochimica et Biophys. Acta (BBA)-Protein Struct. 405, 442-451 (1975).

21. Fawcett, T. An introduction to roc analysis. Pattern recognition letters 27, 861-874 (2006).

22. Sawilowsky, S. S. New effect size rules of thumb. J. Mod. Appl. Stat. Methods 8, 597_599 (2009).

23. Flach, P. A., Hernández-Orallo, J. \& Ramirez, C. F. A coherent interpretation of auc as a measure of aggregated classification performance. In ICML (2011).

24. Nakano, T. et al. Atypical gaze patterns in children and adults with autism spectrum disorders dissociated from developmental changes in gaze behaviour. Proc. Royal Soc. B: Biol. Sci. 277, 2935-2943 (2010).

25. Shiferaw, B. A. et al. Stationary gaze entropy predicts lane departure events in sleep-deprived drivers. Sci. reports $\mathbf{8}$, 1-10 (2018).

26. Lee, P. S. et al. Functional connectivity of the inferior frontal cortex changes with age in children with autism spectrum disorders: a fcmri study of response inhibition. Cereb. Cortex 19, 1787-1794 (2009).

27. Leys, C., Ley, C., Klein, O., Bernard, P. \& Licata, L. Detecting outliers: Do not use standard deviation around the mean, use absolute deviation around the median. J. Exp. Soc. Psychol. 49, 764-766 (2013).

28. Savitzky, A. \& Golay, M. J. Smoothing and differentiation of data by simplified least squares procedures. Anal. chemistry 36, 1627-1639 (1964). 
29. Graham, R. L. \& Yao, F. F. Finding the convex hull of a simple polygon. J. Algorithms 4, 324-331 (1983).

30. Welch, $\mathrm{P}$. The use of fast fourier transform for the estimation of power spectra: a method based on time averaging over short, modified periodograms. IEEE Transactions on audio electroacoustics 15, 70-73 (1967).

31. Shannon, C. E. A mathematical theory of communication. The Bell system technical journal 27, 379-423 (1948).

32. Richman, J. S. \& Moorman, J. R. Physiological time-series analysis using approximate entropy and sample entropy. Am. J. Physiol. Circ. Physiol. 278, H2039-H2049 (2000).

33. Sullivan, G. M. \& Feinn, R. Using effect size—or why the $\mathrm{p}$ value is not enough. J. graduate medical education 4, 279 (2012).

34. Benjamini, Y. \& Hochberg, Y. Controlling the false discovery rate: a practical and powerful approach to multiple testing. J. Royal statistical society: series B (Methodological) 57, 289-300 (1995).

35. Goldberger, J., Hinton, G. E., Roweis, S. \& Salakhutdinov, R. R. Neighbourhood components analysis. Adv. neural information processing systems 17, 513-520 (2004).

\section{Legends}

Figure 1. Extrapolating results of Auto-regressive model using the average of parameters. Gaze extrapolation results using mixed (A), Go positive (C) and negative (E), and NoGo positive (G) and negative (I) coefficients. (B, D, F, H, J) show respectively the extrapolated gaze-to-obj distance and velocity results for mixed (typical-avg: 0.0161, ASD-avg: 0.0156), Go positive (typical-avg: 0.0178, ASD-avg: 0.0165) and negative (typical-avg: 0.0169, ASD-avg: 0.0173), and NoGo positive (typical-avg: 0.0170, ASD-avg: 0.0158) and negative (typical-avg: 0.0141, ASD-avg: 0.0124) coefficients. Solid and dotted green lines represent, respectively, typical children's extrapolated gaze-to-obj distance and the negative of its first derivative (gaze-adjustment velocity) over time. ASD children's extrapolated gaze-to-obj distance and gaze-adjustment velocity are represented by sold and dotted orange lines, respectively.

Figure 2. Star plots depicting four subjects' response-type-gaze and significant spatial features.(A-B) Features representing correctly-classified and misclassified typical children. (C-D) Features representing correctly-classified and misclassified ASD children. Black-line indicates zero values. 13 indexes represent reduced gaze-adjustment features (1-5), velocity-sen, acceleration-avg, fixation-var, distance-sen, angle-sen, gaze-obj-en, gaze-obj-sen, and gaze-obj-spe.

Figure 3. Game interface of the CatChicken system. (A) Nine red flowers representing the locations in which a stimulus can appear; (B) Go and (C) NoGo characters.

Figure 4. Information measured by the CatChicken system. While playing the Go/NoGo game, CatChicken records children's response types and times, and locations of stimulus and gaze over time. The response types are Go-positive (green), NoGopositive (blue), Go-negative (orange), and NoGo-negative (red). The values of object and gaze locations are normalized to range from 0 to 1 .

Figure 5. Experimental protocol of this study. The distance between the child and the monitor was about $60 \mathrm{~cm}$.

Figure 6. Features extraction pipeline used in this study. The inputs consists of gaze and object locations, response, and response time.

Table 1. Significant difference was found in features related to gaze behavior. Statistical significance and effect size of between-group $(p)$ differences of individual spatial features, as measured by Student $t$ and Mann-Whitney $U$ tests. Within-group mean values appear at far right (mean \pm s.d.). $d$ denotes Cohen's effect size measure. Percentage scale is used to express Go positive and negative, and NoGo positive and negative. While, RT, RT-var, fixation-avg, and fixation-var are expressed in miliseconds. * indicates significant $p$-value after controlling false discovery rate at level 0.05 .

Table 2. Greater difference between the groups was observed when separating the gaze-adjustment features based on response type. Statistical significance and effect size of between-group $(p)$ differences of individual gaze-adjustment features, as measured by Student $t$ and Mann-Whitney $U$ tests. $\alpha, \theta_{1}$, and $\theta_{2}$ are the auto-regressive model's constant term, first, and second coefficients, respectively. Within-group mean values appear at far right (mean \pm s.d.). $d$ denotes Cohen's effect size measure. Two analyses using mixed and response-type-separated coefficients. * indicates significant $p$-value after controlling false discovery rate at level 0.05 .

Table 3. The AdaBoost algorithm obtained high performance when employing gaze-related features. Accuracy rate, MCC, and AUC scores of the AdaBoost algorithm with different modality as inputs (mean \pm s.d.). "Significant" refers to features that differ significantly between the groups. "Performance" denotes game performance features that includes positive and negative Go and NoGo responses, response time, and response time variability.

Table 4. False-positive rate of the AdaBoost algorithm was higher than false-negative rate. Confusion matrix of classification results using response-type-gaze and significant spatial features as input of the AdaBoost algorithm. 
Table 5. The list of spatial features extracted from response and gaze behavior. Average was computed with arithmetic mean and variance with standard deviation.

\section{Acknowledgements}

This work was supported by JSPS Kakenhi under Grant No. JP26285212 and JP18H01041. The authors thank Sayaka Hotchi for her assistance in data acquisition.

\section{Author contributions statement}

PP conceived the experiment, developed the game, analyzed the results, and wrote the manuscript. KS conceived the experiment, designed the data analysis method, and designed the game. KJ was involved in designing the experiment and data collection. SA designed the data analysis method, analysed the results and wrote the manuscript. All authors reviewed the manuscript.

\section{Additional information}

Competing interests: The authors declare no competing interests 


\section{Supplementary Files}

This is a list of supplementary files associated with this preprint. Click to download.

- AttentionTest.mp4

- AttentionTestFinalnomark.mp4

- Gaze1.mp4

- SupplementaryMaterials.pdf 